\title{
On the Performance of Push and Pull Models with the Price-Sensitive Demand
}

\author{
Xiaohong Zhang ${ }^{1}$ Jihong $\mathrm{Ou}^{2}$ \\ ${ }^{1}$ School of Politics and Public Administration, Soochow University, Suzhou 215006, P.R.China \\ ${ }^{2}$ NUS Business School,National University of Singapore, Singapore 117592, Singapore
}

\begin{abstract}
In this paper we study a supply chain in which a supplier sells a single product to an independent retailer facing a price-sensitive demand while the sales price is endogenously determined by the retailer, and compare the performance of supply chain between push and pull model. We prove that for the multiplicative form of end-customer demand, at optimality, the wholesale price, order quantity and the retail price in pull model are greater than in push model, respectively. We further demonstrate that the supplier obtains greater profit in pull model than in push model, but it is opposite to the retailer.
\end{abstract}

Keywords: Supply chain management, Push, Pull, Price-sensitive demand

\section{Introduction}

A supply chain consists of independent firms each having his own profit concern, in general, does not achieve optimal performance, i.e., supply chain coordination. However, optimal performance is achievable in a decentralized supply chain if the firms coordinate by contracting on a set of transfer payments such that each member's objective becomes aligned with the supply chain's objective.

In a decentralized supply chain system, each firm's decisions will affect other firms's actions. Then, other firms in the supply chain will react to those actions in a spectrum of ways. In one extreme of this spectrum, the pull model, the distributor would agree to a per-unit price to pay the supplier and then pull as many units of the item as it needed to satisfy demand. The supplier would then order a quantity of the item in anticipation of how many units would be pulled when the distributor responses to the demand realization. Since the supplier would bear the cost of any items that were ordered and were not subsequently pulled by the distributor, he would bear the inventory risk.
At the other end of the spectrum, push model, the suppliers would specify prices for their items, and the distributor would determine the quantities to order. In this case, the distributor would agree to receive whatever quantities she orders regardless of the realization of demand, and she would bear all of the inventory risk. There are two other situations that can be represented by a pull contract: Vendor Managed Inventory(VMI) with consignment inventory(the supplier decides how much inventory to stock at the retailer and owns that inventory), or drop shipping(the supplier holds the inventory and ships directly to consumers, bypassing the retailer). In practice, most supplier-distributor relationships fall somewhere between these two extremes. For example, Dell typically provides at least some assurance as to the minimum quantities of certain specialized components, e.g. a newly released version of CPU. However, for items that are more standard, e.g. memory chips, it is more likely to let suppliers bear the full inventory risk. In fact, it is the lead-time, or the length of time from sending orders to receiving products they ordered, that persuades the supplier or retailer to bear the inventory risk. In this paper, we study a simplified version of supply chain. There is only one order opportunity both in push model and pull model, which occurs well before the selling season due to the long order lead time. The customer demand is price-dependent, stochastic. We consider the push contract and pull contract when the supply chain is composed of only one supplier and one retailer. The rest of the paper is organized as follows. Section 2 contains a brief review of the relevant literature on pull contract and push model of a supply chain as well as on price-sensitive demand. Section 3 describes the basic model when there is single retailer and presents the comparisons of performance under there different scenarios: centralized, pull and push model. Section 4 concludes the paper with a summary of results and discussion of future research directions. 


\section{Brief Review of the Litera- ture}

Our research relates most closely to existing research on wholesale-price-only supply contracts and on price-dependent demand. Following a large amount of research on relatively elaborate coordination mechanisms, e.g. returns policies, quantity flexibility, revenue sharing, etc., Lariviere and Porteus (2001) were the first to formally model a linear wholesale-price-only supply contract. They considered a setting in which the retail price is fixed and the supplier moves first by announcing a wholesale price, then the retailer responds by ordering as a newsvendor. (This setting was later described by Cachon (2004) as a push mode of operations because the inventory is pushed down to the retailer, who bears all of the risk.) Among the more interesting conclusions of Lariviere and Porteus is that the retailer's price sensitivity is increasing in the coefficient of variation for demand, and that the retailer can become worse off as demand variability decreases. It was also shown that, in many settings, a price-only contract achieves a high level of supply chain efficiency, suggesting that more elaborate contractual mechanisms my be unnecessary in many settings.

Cachon and Lariviere (2001) considered a closely related setting in which a two-firm supply chain, here consisting of a supplier and a manufacturer, facing newsvendor demand. However, here it is the downstream firm, i.e. the manufacturer, that moves first to set the contractual terms, and it is the upstream firm that determines the amount to invest in capacity or inventory(This setting was also later described by Cachon (2004) as a pull model of operations because the inventory is pulled up to the upstream firm, who bears all of the risk). They study a number of contractual relationships, but their focus is on how these contracts affect the manufacturer's incentive to accurately report its demand forecast to its supplier.

Cachon (2004) unifies this line of research by considering both the setting in which the upstream firm sets the price, which he characterizes as a push mode of operation, and the setting in which the downstream firm sets the price, which he characterizes as a pull mode of operation. Among the conclusions of this paper is that supply chain efficiency is higher under equilibrium pull than under equilibrium push modes of operation. Taylor (2004) also considers both push and pull modes of operation, to which he refers as early and late sales to the retailer. However, his model is based on price depen- dent demand with an uncertainty parameter, and he focuses on how the retailer's private information about demand or ability to influence demand affect which mode of operation is preferred. Zhang, Ou and Gilbert (2007) study an assemble-to-order environment involving a single product sold in two different configurations, both of which require a unique component stocked in advance and are assembled on the same capacity limited equipment, whose assembly capacity is allocated to maximize system profit for any given product demands and components availability. They first solve for the first-best stocking policy when the components are produced internally. Then they investigate how different forms of contract between the assembler and the component suppliers will affect coordination of the supply chain as well as each party's profit when the components are procured from external suppliers. Finally, they show that it is possible to coordinate the supply chain with a single-price contract between each supplier and the assembler while awarding all parties positive profit.

A distinguishing feature of the above papers is that they all consider the setting in which the retail price is fixed. As noted by Kandel (1996), with the price-dependent demand, it is considerably more complicated, because the retailer should choose his retail price in addition to his order quantity. However, in general, the incentives provided to align one action(e.g., the order quantity) may cause distortions with the other action(e.g., the retail price).Granot and Yin (2005) study the effectiveness of returns policies in the price-dependent newsvendor model. They focus on whether the introduction of buy-back contract can improve the channel efficiency for some expected demand functions.

Some related work has been done on the comparisons of performance of supply chain between push model and push model, e.g. Cachon (2004), Gerchak and Wang (2003) and Wang and Gerchak (2003), etc. However, Cachon (2004) focuses on the allocation of inventory risk among the supply chain's members when the retail price is fixed. Gerchak and Wang (2003) and Wang and Gerchak (2003) compare push and pull modes of operation and show how the relative performance of the two modes depends upon the assembly firm's share of total costs and the number of suppliers. Gurnani and Gerchak (2004) consider a similar framework, but they allow for the suppliers to be subject to random yields.

Although our work also focuses on comparing the performance of supply chain between push 
model and pull model, in contrast to the papers described above, we do not assume a stationary retail price. Instead, we consider a situation where the demand of end-customer market is price-dependent, i.e., the retailer can adjust the demand via its retail price.

\section{Model formulation: Single retailer}

Consider a supply chain, wherein a risk-neutral supplier, who orders/or produces a single product at a fixed marginal cost $c$, sells that product at a wholesale price $w$ per unit to a risk-neutral retailer, who then sells it at a marginal retail price $p$ to a end-customer market, over a single season. We assume in this paper that demand of the retailer during the selling season is stochastic with a multiplicative form $D=D(p) \epsilon, D(p)$ is the expected amount of demand $D$, which decreases in the retail price $p$, and $\epsilon(\epsilon \geq 0)$ is the random part of demand $D$, which is a positive random variable with mean normalized to 1 . The multiplicative demand model, which is commonly used in the economics and operations management literature, was initially proposed by Karlin and Carr (1962), and it could induce a constant coefficient of variation for any retail price while the variance of demand increases with expected demand. $D$ has a density function satisfying $f(x \mid p)=\frac{1}{D(p)} f_{\epsilon}\left(\frac{x}{D(p)}\right)$, for $x \geq 0$, where $f_{\epsilon}()$ is the density of a positive random variable with mean 1 . For the purpose of gaining some insights into the dynamics of the problem, throughout this paper, we adopt the same assumption as Emmons and Gilbert (1998) had made that $f_{\epsilon}(x)$ is a uniform distribution on the interval $[0,2]$, i.e., the density function is of the form

$$
f_{\epsilon}(x)=\left\{\begin{array}{cc}
\frac{1}{2} & x \in[0,2] \\
0 & \text { otherwise }
\end{array}\right.
$$

We further note that, for any retail price $p$, the highest demand from the end-customer market is $2 D(p)$, because it is easy to note that the Cumulative Distribution Function(CDF) of demand is

$$
F_{D}(x)= \begin{cases}0 & x<0 \\ \frac{1}{2 D(p)} x & x \in[0,2 D(p)] \\ 1 & x>2 D(p)\end{cases}
$$

Although our choice of the uniform distribution is somewhat arbitrary, it is easy for a manager to visualize and it allows us to illustrate the dynamics of the problem. To keep things as simple as possible, let us assume that demand is linear, and is of the form: $D(p)=a(p-k)$, where $a<0$ and $k>0$ are constants. It is easy to note that when $p=k$, the expected profits of both supplier and retailer vanish, since the expected demand from the external market is zero. We also assume that unsatisfied demand is lost without any penalty costs for lost sales, and that units remaining at the end of the season are salvaged for $v$ per unit, $v<c$, no matter which firm, supplier or retailer, salvages the unit. Thus, we assume for feasibility that $0 \leq v<c \leq w \leq p<k$ in the sequel, except as otherwise noted. We have chosen a linear demand model for its simplicity and because it is commonly used in the literature. Several authors, including Chu and Messinger (1993), Riordan (1985), and Bagwell and Ramey (1990), have modelled uncertain demand as a linear function of price in which the intercept is a random variable.

\subsection{Integrated Supply Chain}

To address the impact of push model and pull model to the performance of decentralized supply chain, we first present and analyze a version of this supply chain system under a centralized decision maker, i.e., the integrated supply chain, as a benchmark. The integrated supply chain maximizes the sum of the retailer's profit and the supplier's profit. The supply chain's decisions are both the production quantity $q$ and the sales price $p(p<k)$. The supply chain's expected profit is

$$
\begin{aligned}
\Pi(q, p)= & E_{D}\left[p \min \{q, D\}+v(q-D)^{+}-c q\right] \\
= & (p-c) q-(p-v) \int_{0}^{q} F_{D}(x) \mathrm{d} x \\
= & (p-c) q-(p-v)\left[q F_{\epsilon}\left(\frac{q}{D(p)}\right)\right. \\
& \left.-\int_{0}^{\frac{q}{D(p)}} D(p) x f_{\epsilon}(x) \mathrm{d} x\right] \\
= & (p-c) q-\frac{(p-v) q^{2}}{4 a(p-k)}
\end{aligned}
$$

Notice from (3) that $\pi(q, p)$ is concave in $q$ for a given $p$. Thus, it is possible to reduce the problem $\max _{q, p} \pi(q, p)$ to an optimization problem over the single variable $p$ by first solving for the optimal value of $q$ as a function of $p$ and then substituting the result back into $\pi(q, p)$. To my knowledge, this method was primarily introduced by Whitin (1955). Hence, we obtain the optimal values of retail price and production quantity as follows:

$$
p^{0}=\frac{k+3 v+\sqrt{(k-v)(k-9 v+8 c)}}{4}
$$


and

$$
\begin{aligned}
q^{0} & =\frac{2 a\left(p^{0}-k\right)\left(p^{0}-c\right)}{p^{0}-v} \\
& =\frac{a}{2}[9 v-5 k-4 c+3 \sqrt{(k-v)(k-9 v+8 c)}]
\end{aligned}
$$

For notational convenience, let $\Pi^{0}=\Pi\left(q^{0}, p^{0}\right)$. We substitute $p^{0}$ and $q^{0}$ into the expected integrated supply chain profit function (3), and simplify it to

$$
\begin{aligned}
\Pi^{0} & =\frac{a}{32(v-c)}(\sqrt{(k-v)(k-9 v+8 c)}-k+3 v-2 c) \\
& (k+3 v-4 c+\sqrt{(k-v)(k-9 v+8 c)})^{2}
\end{aligned}
$$

\subsection{Push Model}

Now, we turn to a push model, wherein a supplier sells the products which he has purchased or produced in advance to a retailer, who then resell them to end-customer market. The sequence of events is as follows. First, the supplier acts as the Stackelberg leader by announcing the wholesale price $w$ per unit, at which products will be sold to retailer before the selling season. In response to the proposed $w$, the retailer, who faces a newsvendor decision with price-dependent demand, commits to an order quantity $q$ prior to the selling season(at this time, the inventory is in fact pushed down to the retailer and the retailer bears all of the risk while the supplier has no inventory risk), and a retail price $p(p \geq w)$, at which to sell those products during the season. Next, the supplier orders from its outer suppliers or produces the $q$ quantity of products and deliver them to the retailer before the season. Thereafter, demand is realized and any unsatisfied demands are lost without any penalty cost. At the end of the season, all the remaining products are salvaged(by the retailer) at a marginal price $v$ and all costs and revenues are incurred.

By replacing the sign $c$ with $w$ in (3), we can easily derive the retailer's profit

$$
\hat{\pi}_{r}=(p-\hat{w}) q-\frac{(p-v) q^{2}}{4 a(p-k)}
$$

When $\hat{w}$ is given, we obtain the unique optimal order quantity by the similar way above

$$
\hat{q}=\frac{a}{-4}(\sqrt{k-9 v+8 \hat{w}}-3 \sqrt{k-v})^{2}
$$

and the optimal retail price

$$
\hat{p}=\frac{k+3 v+\sqrt{(k-v)(k-9 v+8 \hat{w})}}{4}
$$

Taking the retailer's reaction functions into account, the supplier's profit is

$$
\begin{aligned}
\hat{\pi}_{s}(\hat{w}) & =\hat{\pi}_{s}(\hat{q}, \hat{w}) \\
& =(\hat{w}-c) \hat{q} \\
& =\frac{a}{2}(\hat{w}-c)(9 v-5 k-4 \hat{w} \\
& +3 \sqrt{(k-v)(k-9 v+8 \hat{w})}) \\
& =\frac{a}{-4}(\hat{w}-c)(\sqrt{k-9 v+8 \hat{w}}-3 \sqrt{k-v})^{2}
\end{aligned}
$$

Notice that the optimal solution of optimization problem $\max _{\hat{w} \geq c} \hat{\pi}_{s}(\hat{w})$ is the same as the optimization problem $\max _{\hat{w} \geq c} \log \left(\hat{\pi}_{s}(\hat{w})\right)$ through the monotonicity property of function $\log (x)$ for $x>0$, we can compute the optimal wholesale price

$\hat{w}=\frac{5 k+32 c+27 v+3 \sqrt{(k-v)(17 k-81 v+64 c)}}{64}$

for it satisfies that $\hat{w} \geq c$. By substituting (11) into (8) and (9), we obtain the optimal values of $p$ and $q$ in the corresponding push model

$$
\hat{p}=\frac{7 k+9 v+\sqrt{(k-v)(17 k-81 v+64 c)}}{16}
$$

and

$$
\hat{q}=\frac{-a}{32}[49 k+32 c-81 v-9 \sqrt{(k-v)(17 k-81 v+64 c)}]
$$

\subsection{Pull Model}

In contrast to push model, the decision sequence in a pull model is as follows. First, the retailer, who really acts like a Stackelberg leader, announces its preferred marginal wholesale price $w$, at which the retailer will purchase the products from the supplier during the selling season, after having made its price decisions in mind. In response to this proposed $w$, the supplier, who faces a newsvendor decision, commits to an order quantity $q$ prior to the season(as a matter of fact, the supplier pulls the inventory up to him and bears all of the risk at this time). Next, the supplier's upstream suppliers deliver the product to the supplier at the start of selling season. Thereafter, demand is realized and any unsatisfied demands are lost without any penalty cost. At the end of the season, all the remaining products are salvaged(by the supplier) at a marginal price $v$ and all costs and revenues are incurred. 
The supplier's expected profit is

$$
\begin{aligned}
\pi_{s}(q, w) & =E_{D}\left[w \min \{q, D\}+v(q-D)^{+}-c q\right] \\
& =(w-c) q-(w-v) \int_{0}^{q} F_{D}(x) \mathrm{d} x \\
& =(w-c) q-(w-v) \frac{q^{2}}{4 a(p-k)}
\end{aligned}
$$

Because $\pi_{s}(q, w)$ is strictly concave in $q$, the supplier's optimal quantity is implicitly defined by

$$
F_{D}(q)=\frac{w-c}{w-v}
$$

There is a one-to-one relationship between the production quantity $q$ and the wholesale price $w$, that is, $q=2 D(p) \frac{w-c}{w-v}=2 a(p-k) \frac{w-c}{w-v}$, or $w=$ $\frac{2 a c(p-k)-q v}{2 a(p-k)-q}$. In addition, it follows that the production quantity $q$ is increasing with respect to wholesale price $w$ through the fact that $\frac{\mathrm{d} q}{\mathrm{~d} w}=$ $2 a(p-k) \frac{c-v}{(w-v)^{2}}>0$. Certainly, the optimal value of $q$ satisfies that $0 \leq q \leq 2 D(p)$.

The expected profit of retailer is

$$
\begin{aligned}
\pi_{r}(q, p) & =E_{D}[(p-w) \min \{q, D\}] \\
& =(p-w)\left[q-\int_{0}^{q} F_{D}(x) \mathrm{d} x\right] \\
& =2 a(p-w)(p-k)\left[1-\frac{w-c}{2(w-v)}\right] \frac{w-c}{w-v}
\end{aligned}
$$

The optimal retail price is

$$
p^{*}=\frac{w+k}{2}
$$

Substituting $p^{*}$ into $q=2 a(p-k) \frac{w-c}{w-v}$, we obtain the optimal order quantity

$$
q^{*}=a \frac{(w-k)(w-c)}{w-v}
$$

Thus, using (18) we can rewrite the supplier's expected profit in terms of $w$ :

$$
\pi_{s}(w)=\frac{-a}{2} \frac{(k-w)(w-c)^{2}}{w-v}
$$

Through $\frac{\mathrm{d} \pi_{s}(w)}{\mathrm{d} w}=0$, we obtain the optimal wholesale price

$$
w^{*}=\frac{3 v+k}{4}+\frac{1}{2} \sqrt{\frac{(v-k)^{2}}{4}-2(c-v)(v-k)}
$$

for it satisfies that $w^{*} \geq c$. By substituting (20) into (17) and (18), we obtain the optimal values of $p$ and $q$ in the corresponding pull model

$$
p^{*}=\frac{5 k+3 v+\sqrt{(k-v)(k-9 v+8 c)}}{8}
$$

and

$$
q^{*}=\frac{-a}{4}[5 k+4 c-9 v-3 \sqrt{(k-v)(k-9 v+8 c)}]
$$

The retailer's expected profit is:

$$
\pi_{r}(w)=-\frac{a}{2}(w-k)^{2}\left(1-\frac{1}{2} \frac{w-c}{w-v}\right) \frac{w-c}{w-v}
$$

\subsection{Performance Comparison between Push Model and Pull Model}

PROPOSITION 1: In the above integrated supply chain as well as decentralized supply chain with push or pull contract:

(i) $p^{*} \geq \hat{p} \geq p^{0}$

(ii) $q^{0}>q^{*} \geq \hat{q}$;

(iii) $\hat{w} \leq w^{*}$.

It follows from Proposition 1 that, as expected, the integrated supply chain would be preferred by the end customers to a decentralized supply chain both with pull and push strategies, in the sense that it offers a lower retail price and makes a larger amount of products available to customers. This coincides with the well-studied Double Marginalization phenomenon. And it is easy to understand that there always exists conflict of profit-pursuit between the supplier and the retailer no matter what contract has been signed. But, there is something very interesting and it is what we intended to do the comparison between the pull and push model. From Proposition 1, it also follows that both the retail price and the quantity available for the end customers with pull model are higher than that with push model while the wholesale price with pull is higher than that with push. We note that the results derived in Proposition 1 are consistent with those derived by Cachon(2004) for his demand model.

Further, having the Proposition 1, we derive the following conclusion.

PROPOSITION 2:

(i) The supplier's maximum profit with pull is greater than with push, i.e., $\pi_{s} \geq \hat{\pi}_{s}$;

(ii) The retailer's maximum profit with push is greater than with pull, i.e., $\hat{\pi}_{r} \geq \pi_{r}$;

(iii) The system's maximum profit with pull is greater than with push, i.e., $\pi \geq \hat{\pi}$, or, $\pi_{r}+\pi_{s} \geq$ $\hat{\pi}_{r}+\hat{\pi}_{s}$

(iv) The total expected profit of centralized system is greater than the total expected profit in pull mode, i.e., $\Pi^{0} \geq \pi$.

Proposition 2 indicates that supplier prefer the pull 
mode over the push mode while the retailer prefers the push mode over the pull mode. Thus, assuming an optimal contract is chosen, a firm, the retailer or the supplier, always earns a higher profit if the firm bears the supply chain's inventory risk. In other words, counter to intuition, a firm should not negotiate with the objective of getting the other form to bear more inventory risk. We also note that the results derived in Proposition 2 are consistent with those derived by Cachon(2004) for his demand model. More importantly, the supply chain relative to the pull mode is attractive for system managers to the push mode.

\section{Conclusions and future re- search}

In this paper we study a supply chain in which a supplier sells a single product to an independent retailer facing a price-sensitive demand while the sales price is endogenously determined by the retailer, and compare the performance of supply chain between push and pull model. We demonstrate that the wholesale price, order quantity and the retail price in pull model are greater than in push model at optimality, respectively. We further demonstrate that the supplier obtains greater profit in pull model than in push model, but it is opposite to the retailer. In other words, the results show that the allocation of inventory risk matters for supply chain efficiency even if firms are risk neutral. For example, the whole supply chain's profit in a pull model, in which the supplier bears the inventory risk and the retailer has chosen her optimal purchasing price, is greater than that in a push model, in which the retailer bears the inventory risk and the supplier has chosen her optimal wholesale price. This result is very interesting not only for academic researchers but also for practical managers. For it provides us several different choices.

Our analysis in this paper focuses on the situation when the end-customer demand follows a multiplicative form. It is also interesting to study the case where the end-customer demand is additive form. Further, are these results also true when there exist several competing retailers? We will continue our investigation in all these directions.

\section{References}

[1] S. Karlin and C. R. Carr, Integration of Visual Modules,Academic Press,New York,1989.

[2] S. Karlin and C. R. Carr, Prices and Optimal Inventory Policy. In K. J. Arrow, S. Karlin and H. Scarf, editors, Studies in Applied Probability and Management Science,pp. 159-172, Stanford University Press, Stanford, California, 1962.

[3] M. L. Braunstein,Motion and texture as sources of slant information. Journal of Experimental Psychology,78:247-253, 1968.

[4] G. P. Cachon, The allocation of inventory risk in a supply chain: Push, pull, and advancepurchase discount contracts. Management Science, 50:222-238, 2004.

[5] G. P. Cachon and M. A. Lariviere, Contracting to assure supply: How to share demand forecasts in a supply chain. Management Science, 47: 629-646, 2001.

[6] Y. Wang and Y. Gerchak, Capacity games in assembly systems with uncertain demand. Manufacturing and Service Operations Management, 5: 252-267, 2003.

[7] Y. Gerchak and Y. Wang, Revenue sharing vs. wholesale-price contracts in assembly systems with random demand. Production and Operations Management, 13: 23-33, 2003.

[8] M. A. Lariviere and E. Porteus, Selling to the newsvendor: An analysis of price-only contracts. Manufacturing and Service Operations Management, 3: 293-305, 2001.

[9] E. Kandel, The Right to Return. Journal of Law and Economics, 39: 329-356, 1996.

[10] D. Granot and S. Yin, On the effectiveness of returns policies in the price-dependent newsvendor model. Naval Research Logistics, 52: 765$779,2005$.

[11] X. Zhang, J. Ou and S. M. Gilbert, Coordination of Stocking Decisions in an Assemble to Order Environment. Forthcoming in European Journal of Operational Research, 2007.

[12] T. M. Whitin, Inventory Control and Price Theory. Management Science, 2: 61-68, 1955.

[13] H. Emmons and S. M. Gilbert, Note. The role of returns policies in pricing and inventory decisions for catalogue goods. Management Science, 44: 276-283, 1998.

[14] M. Riordan, Imperfect information and dynamic conjectural variations. The RAND Journal of Economics, 16: 41-50, 1985.

[15] K. Bagwell and G. Ramey, Advertising and pricing to deter or accommodate entry when demand is unknown. International Journal of 
Industrial Organization, 8: 93-113, 1990.

[16] W. Chu and P. R. Messinger, Product proliferation, slotting allowances, and information: Sources of retailer clout. Technical Report, MIT, Cambridgs, MA, 1993.

\section{APPENDIX}

\section{PROOF OF PROPOSITION 1:}

(i) $p^{*} \geq \hat{p} \geq p^{0}$. It follows that $3 \sqrt{k-v}+$ $2 \sqrt{k-9 v+8 c} \geq \sqrt{17 k-81 v+64 c}$ through the fact that $(3 \sqrt{k-v}+2 \sqrt{k-9 v+8 c})^{2}-$ $(\sqrt{17 k-81 v+64 c})^{2}=\frac{32 \sqrt{k-9 v+8 c}(k-c)}{3 \sqrt{k-v}+\sqrt{k-9 v+8 c}} \geq 0$. Thus it is easy to verify that

$$
\begin{aligned}
p^{*}-\hat{p} & =\frac{\sqrt{k-v}}{16}[3 \sqrt{k-v}+2 \sqrt{k-9 v+8 c} \\
& -\sqrt{17 k-81 v+64 c}] \\
& \geq 0
\end{aligned}
$$

that is, $p^{*} \geq \hat{p}$.

Similarly, we can easily verify that $3 \sqrt{k-v}+\sqrt{17 k-81 v+64 c} \geq 4 \sqrt{k-9 v+8 c}$ through the fact that $(3 \sqrt{k-v}+$ $\sqrt{17 k-81 v+64 c})^{2}-(4 \sqrt{k-9 v+8 c})^{2}=$ $(9 \sqrt{k-v}-\sqrt{17 k-81 v+64 c})(3 \sqrt{k-v}+$ $\sqrt{17 k-81 v+64 c}) \geq 0$. Thus it follows that

$$
\begin{aligned}
\hat{p}-p^{0} & =\frac{1}{16}[3 \sqrt{k-v}+\sqrt{17 k-81 v+64 c} \\
& -4 \sqrt{k-9 v+8 c}] \\
& \geq 0
\end{aligned}
$$

that is, $\hat{p} \geq p^{0}$.

(ii) $q^{0}>q^{*} \geq \hat{q}$. It follows that $3 \sqrt{k-v}+8 \sqrt{k-9 v+8 c} \leq 3 \sqrt{17 k-81 v+64 c}$ through the fact that $[3(\sqrt{17 k-81 v+64 c}-$ $\sqrt{k-v})]^{2}-(8 \sqrt{k-9 v+8 c})^{2}=(9 \sqrt{k-v}-$ $\sqrt{17 k-81 v+64 c})^{2} \geq 0$. It is easy to verify that

$$
\begin{aligned}
q^{*}-\hat{q} & =\frac{3 a}{32}[3 \sqrt{k-v}+8 \sqrt{k-9 v+8 c} \\
& -3 \sqrt{17 k-81 v+64 c}] \\
& \geq 0
\end{aligned}
$$

that is, $q^{*} \geq \hat{q}$.

Note that $9 v-5 k-4 c+3 \sqrt{(k-v)(k-9 v+8 c)}<0$ since $v<c<k$. It is easy to verify that

$$
\begin{aligned}
q^{0}-q^{*} & =\frac{a}{2}[9 v-5 k-4 c+3 \sqrt{(k-v)(k-9 v+8 c)}] \\
& -\frac{-a}{4}[9 v-5 k-4 c+3 \sqrt{(k-v)(k-9 v+8 c)}] \\
& =\frac{a}{4}[9 v-5 k-4 c+3 \sqrt{(k-v)(k-9 v+8 c)}] \\
& >0
\end{aligned}
$$

that is, $q^{0}>q^{*}$.

(iii) $\hat{w} \leq w^{*}$. Let us denote $M$ by $M=\frac{c-v}{k-v}$. It is obvious that $0 \leq M \leq 1$. We note that, for arbitrary $M \in[0,1]$, the following inequality holds

$$
-11+32 M+3 \sqrt{17+64 M}-16 \sqrt{1+8 M} \leq 0
$$

Thus, from (11) and (20), it follows that

$$
\begin{aligned}
\hat{w}-w^{*} & =\frac{1}{64}[32 c-11 k-21 v \\
& +3 \sqrt{(k-v)(17 k-81 v+64 c)} \\
& -16 \sqrt{(k-v)(k-9 v+8 c)}] \\
& =\frac{k-v}{64}[-11+32 M+3 \sqrt{17+64 M} \\
& -16 \sqrt{1+8 M}] \\
& \leq 0
\end{aligned}
$$

that is, $\hat{w} \leq w^{*}$

PROOF OF PROPOSITION 2: Let us denote $N$ and $Q$ by $N=k-c$ and $Q=c-v$. Note that $N \geq 0$ and $Q \geq 0$ since $v<c<k$. We also define that $A 0=\sqrt{(k-v)(k-9 v+8 c)}$, or $A 0=\sqrt{(N+Q)(N+9 Q)}$, as well as that $B 0=\sqrt{(k-v)(17 k-81 v+64 c)}$, or $B 0=$ $\sqrt{(N+Q)(17 N+81 Q)}$.

(i) $\pi_{s} \geq \hat{\pi}_{s}$

We substitute $p^{*}$ and $q^{*}$ into the expected profit function of supplier on pull mode, $\pi_{s}=$ $\frac{-a}{2} \frac{(k-w)(w-c)^{2}}{w-v}$, and simplify it to

$$
\pi_{s}=\frac{-a}{32}\left[2 N^{2}-36 N Q-54 Q^{2}+2(N+9 Q) A 0\right]
$$

Similarly, by substituting $\hat{w}$ and $\hat{q}$ into the expected profit function of supplier on push mode, $\hat{\pi}_{s}=(\hat{w}-$ c) $\hat{q}$, we can simplify it to

$$
\begin{aligned}
\hat{\pi}_{s} & =\frac{-a}{32} \frac{1}{32}\left[-107 N^{2}-1782 N Q-2187 Q^{2}\right. \\
& +(51 N+243 Q) B 0]
\end{aligned}
$$

Thus, it follows that

$$
\begin{aligned}
\pi_{s}-\hat{\pi}_{s} & =\frac{-a}{32} \frac{1}{32}\left[171 N^{2}+630 N Q+459 Q^{2}\right. \\
& -(51 N+243 Q) B 0+64(N+9 Q) A 0] \\
& =\frac{-a}{32} \frac{1}{32 Q^{2}}\left[171\left(\frac{N}{Q}\right)^{2}+630 \frac{N}{Q}+459\right. \\
& -\left(51 \frac{N}{Q}+243\right) \sqrt{\left(\frac{N}{Q}+1\right)\left(17 \frac{N}{Q}+81\right)} \\
& \left.+64\left(\frac{N}{Q}+9\right) \sqrt{\left(\frac{N}{Q}+1\right)\left(\frac{N}{Q}+9\right)}\right] \\
& \geq 0
\end{aligned}
$$

where the last inequality is due to the fact that $64(x+9)^{\frac{3}{2}}-27(x+1)^{\frac{3}{2}}-\frac{23 x+567}{3}(17 x+81)^{\frac{1}{2}}>0$ holds for arbitrary $x \geq 0$. that is, $\pi_{s} \geq \hat{\pi}_{s}$.

(ii) $\pi_{r} \leq \hat{\pi}_{r}$

The expected profit of retailer on pull mode is

$$
\begin{aligned}
\pi_{r} & =\frac{-a}{2} \frac{(k-w)(w-c)^{2}}{w-v} \\
& =\frac{-a}{32}\left[N^{2}-18 N Q-27 Q^{2}+(N+9 Q) A 0\right]
\end{aligned}
$$


The expected profit of retailer on push mode is

$$
\begin{aligned}
\hat{\pi}_{r} & =\frac{-a}{32} \frac{1}{64}\left[487 N^{2}+270 N Q-729 Q^{2}\right. \\
& -(79 N-81 Q) B 0]]
\end{aligned}
$$

Thus, it follows that

$$
\begin{aligned}
\hat{\pi}_{r}-\pi_{r} & =\frac{-a}{32} \frac{1}{64}\left[423 N^{2}+1422 N Q+999 Q^{2}\right. \\
& -(79 N-81 Q) B 0-64(N+9 Q) A 0] \\
& =\frac{-a}{32} \frac{1}{64 Q^{2}} \frac{1}{\sqrt{\frac{N}{Q}+1}}\left[\left(423 \frac{N}{Q}+999\right) \sqrt{\frac{N}{Q}+1}\right. \\
& -\left(79 \frac{N}{Q}-81\right) \sqrt{17 \frac{N}{Q}+81} \\
& -64\left(\frac{N}{Q}+9\right) \sqrt{\frac{N}{Q}+9}
\end{aligned}
$$

It is easy to verify that, $(423 x+999) \sqrt{x+1}-(79 x-$ 81) $\sqrt{17 x+81}-64(x+9) \sqrt{x+9} \geq 0$ holds for arbitrary $x \geq 0$. Thus, $\hat{\pi}_{r}-\pi_{r} \geq 0$, that is, $\hat{\pi}_{r} \geq \pi_{r}$. (iii) $\pi \geq \hat{\pi}$.

The total expected profit in pull mode is as follows

$$
\begin{aligned}
\pi & =\pi_{r}+\pi_{s} \\
& =\frac{-a}{2}(k-w)^{2}\left(1-\frac{1}{2} \frac{w-c}{w-v}\right) \frac{w-c}{w-v}+\frac{-a}{2} \frac{(k-w)(w-c)^{2}}{w-v} \\
& =\frac{-3 a}{32}\left[N^{2}-18 N Q-27 Q^{2}+(N+9 Q) A 0\right]
\end{aligned}
$$

The expected profit in push mode is as follows

$$
\begin{aligned}
\hat{\pi} & =\hat{\pi}_{r}+\hat{\pi}_{s} \\
& =(\hat{p}-c) \hat{q}-\frac{(\hat{p}-v) \hat{q}^{2}}{4 a(\hat{p}-k)}+(\hat{w}-c) \hat{q} \\
& =\frac{-3 a}{32}\left(N^{2}-18 N Q-27 Q^{2}+(N+9 Q) A 0\right. \\
& +\frac{-3 a}{32}\left[\frac{27}{64}(N+Q)^{2}+\frac{23 N+567 Q}{192} B 0-(N+9 Q) A 0\right] \\
& =\pi+\frac{-3 a}{32}\left[\frac{27}{64}(N+Q)^{2}\right. \\
& \left.+\frac{23 N+567 Q}{192} B 0-(N+9 Q) A 0\right] \\
& =\pi+\frac{-3 a}{\sqrt{N+Q}}\left[27(\sqrt{N+Q})^{3}\right. \\
& \left.-64(\sqrt{N+9 Q})^{3}+\frac{23 N+567 Q}{3} \sqrt{17 N+81 Q}\right] \\
& =\pi+\frac{-3 a}{32} \frac{\sqrt{N+Q}}{64} Q^{\frac{3}{2}}\left[27\left(\sqrt{\frac{N}{Q}+1}\right)^{3}\right. \\
& \left.-64\left(\sqrt{\frac{N}{Q}+9}\right)^{3}+\frac{23 \frac{N}{Q}+567}{3} \sqrt{17 \frac{N}{Q}+81}\right] \\
& <\pi
\end{aligned}
$$

where the last inequality is based on the fact that $27(x+1)^{\frac{3}{2}}-64(x+9)^{\frac{3}{2}}+\frac{23 x+567}{3}(17 x+81)^{\frac{1}{2}}<0$ since $x>0$.

(iv) $\Pi^{0} \geq \pi$.

The total expected profit of centralized system is:

$$
\begin{aligned}
\Pi^{0} & =a\left(1+\frac{v-k}{p^{0}-v}\right)\left(p^{0}-c\right)^{2} \\
& =\frac{-a}{32} \frac{1}{c-v}[\sqrt{(k-v)(k-9 v+8 c)}-k+3 v-2 c] \\
& {[k+3 v-4 c+\sqrt{(k-v)(k-9 v+8 c)}]^{2} } \\
& =\frac{-a}{32 Q}(A 0-N-3 Q)(N-3 Q+A 0)^{2} \\
& =\frac{-4 a}{32 Q}\left[N^{2}-18 N Q-27 Q^{2}+(N+9 Q) A 0\right] \\
& =\frac{4}{3} \pi \\
& >\pi
\end{aligned}
$$

